\title{
Theranostics
}

Short Research Communication

2012; 2(2):207-214. doi: 10.7150/thno.3806

\section{In Vivo Monitoring of Liver Damage Using Caspase-3 Probe}

\author{
Michitaka Ozaki', Sanae Haga ${ }^{1,2}$, Takeaki Ozawa ${ }^{3 凶}$ \\ 1. Department of Molecular Surgery, Hokkaido University School of Medicine, N-15, W-7, Kita-ku, Sapporo, Hokkaido \\ 060-8638, Japan; \\ 2. The Japan Society for the Promotion of Science (JSPS), Tokyo, Japan; \\ 3. Department of Chemistry, School of Science, The University of Tokyo, 7-3-1 Hongo, Bunkyo-ku, Tokyo 113-0033, Japan.
}

Corresponding author: T.O. (ozawa@chem.s.u-tokyo.ac.jp).

(c) Ivyspring International Publisher. This is an open-access article distributed under the terms of the Creative Commons License (http:/ / creativecommons.org/ licenses/by-nc-nd/3.0/). Reproduction is permitted for personal, noncommercial use, provided that the article is in whole, unmodified, and properly cited.

Received: 2011.11.15; Accepted: 2011.12.24; Published: 2012.02.15

\begin{abstract}
Real-time monitoring of cellular and organ conditions improves our understanding of various physiopathological phenomena. Such monitoring is expected to provide important alternatives for clinical diagnosis and therapy. We have sought to show physiopathological changes of organs as well as cells. Here, we present an example of in vivo imaging of liver states using the luciferase-based caspase-3 optical probe. We examined dynamic changes of apoptosis (caspase-3 activity) of a mouse liver as well as those of liver cells, proving that the emitted signals reflected the biochemically evaluated apoptotic cell death. In live liver cell (AML I2) experiments, the optical probe for caspase-3 activity emitted signals in response to Fas-ligand, staurosporine and hypoxia/reoxygenation, demonstrating that the probe can measure cellular apoptosis quantitatively. We therefore applied this probe for mouse liver ische$\mathrm{mia} /$ reperfusion (I/R) and drug-toxicity to liver. By expressing the probe in a mouse liver adenovirally, we imaged liver caspase-3 activity (i.e. apoptotic damage) non-invasively and chronologically in the hepatic I/R model of mice. The duration of liver ischemia affected the post-ischemic caspase-dependent damage. Ischemia (up to $60 \mathrm{~min}$ ) enhanced liver damage after reperfusion, but prolonged ischemia ( $90 \mathrm{~min}$ of ischemia) induced not apoptotic cell death but necrotic cell death. Direct observations of the changes of organ conditions elucidated the dynamism of organ function and damage. These technologies clearly possess clinical relevance. They are expected to provide a new diagnostic tool for various clinical settings in the future.
\end{abstract}

Key words: imaging, non-invasive monitoring, optical probe, bioluminescence, luciferase.

\section{Introduction}

Real-time monitoring of cellular and organ conditions engenders better understanding of various phenomena. Such technologies are also expected to provide useful information as well as diagnostic and therapeutic options in various clinical settings. Many optical probes have been developed to visualize protease activities for in vivo physiopathological phenomena imaging [1-7]. They have been used to analyze the dynamic changes of cellular and organ damage, including hypoxia/reoxygenation (H/R) of cells and ischemia and reperfusion $(\mathrm{I} / \mathrm{R})$ of organs.

Especially, I/R injury is an important concern in various clinical circumstances including organ transplantation, myocardial infarction, and stroke. In these clinical situations, prolonged ischemia followed by reperfusion results in extended organ apoptosis/necrosis and organ failure [8]. Although the mechanisms of I/R-induced tissue damage are com- 
plex, post-I/R apoptotic damage plays a pivotal role in post-I/R organ failure $[9,10]$. Therefore, non-invasive monitoring of caspase- 3 activity in vivo is informative and is likely to provide important therapeutic information.

We previously developed a novel probe (pcFluc-DEVD) of cyclic luciferase reflecting caspase-3 activity [2]. Two fragments of DnaE inteins are fused to neighboring $\mathrm{N}$-terminal and $\mathrm{C}$-terminal ends of firefly luciferase connected with a substrate sequence of caspase-3 (DEVD) (Fig. 1a). After translation into a single polypeptide, the $\mathrm{N}$-terminal and C-terminal ends of luciferase are ligated by protein splicing, producing a closed circular polypeptide chain. The cyclic luciferase structure is distorted. Therefore, the luciferase loses its bioluminescence activity (inactive form). Once caspase-3 is activated in cells (DEVD is cleaved), Fluc changes into an active form if the substrate sequence is digested using the protease, thereby restoring luminescence activity (Fig. $1 b)$.

Applicability of the cyclic luciferase was first demonstrated for quantitative detection of the caspase-3 activities in living cells. The probes were expressed in HeLa cells using a conventional plasmid transfection technique and stimulated with stauro- sporine. Cell-based analysis using the cyclic luciferase allowed for precise and quantitative measurements of caspase- 3 activities because it enabled analysis of a statistically significant number of cells in a single assay format. The response of cyclic luciferase upon caspase- 3 activation was extremely rapid, suggesting high-throughput screening and characterization of therapeutic anticancer drugs and caspase inhibitors. Moreover, the cyclic luciferase enabled in vivo real-time imaging of caspase- 3 activities in living mice. Chemical compounds, in many cases, are metabolized or chemically modified in living mice. Effective concentrations of the chemical compounds can be estimated noninvasively using this imaging method.

Here, we present data related to in vitro measurement of caspase-3 activity of live liver cells challenged by Fas-ligand (FasL), staurosporine (STS), and hypoxia, and also in vivo imaging of caspase-3 activity in post-ischemic liver using the caspase-3 probe. By transfecting adenovirus vectors encoding pcFluc-DEVD (AdpcFluc), we investigated whether these probes will monitor redox states and apoptosis in live cells and liver during hypoxia/reoxygenation $(\mathrm{H} / \mathrm{R})$ and $\mathrm{I} / \mathrm{R}$ in mice. We confirmed the efficacy of these probes in a mouse model and showed their potential as clinical tools for additional applications.

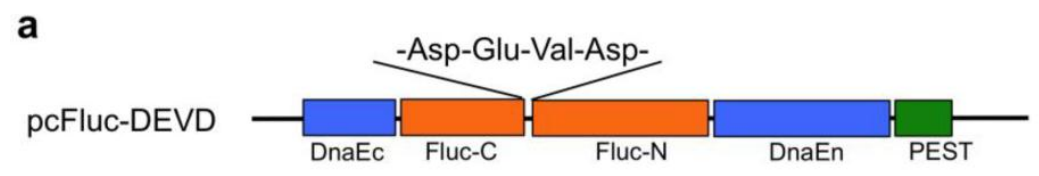

b

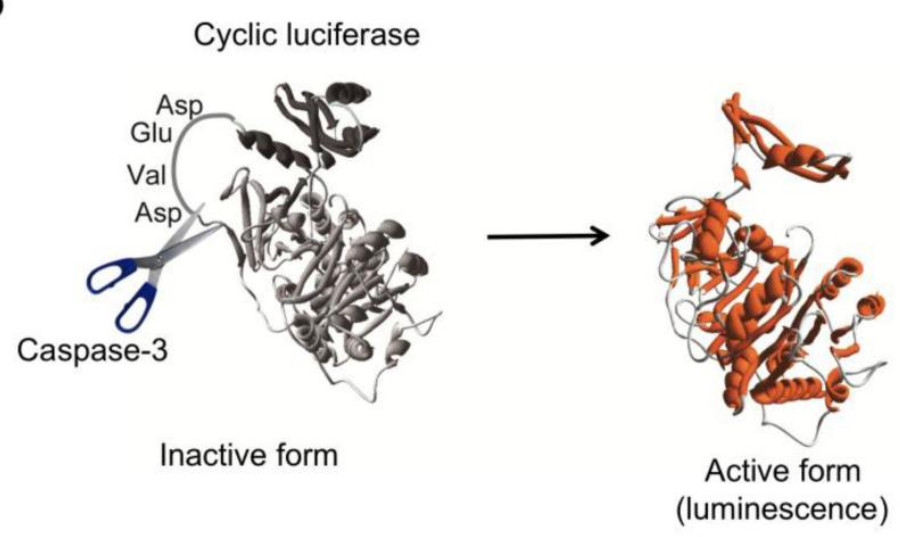

Figure I. (a) Schematic structures of cDNA constructs. Fluc- $\mathrm{N}$ and Fluc- $\mathrm{C}$ indicate $\mathrm{N}$-terminal and $\mathrm{C}$-terminal fragments of Fluc. The Flanking sides of the luciferase are connected with $\mathrm{C}$-terminal and $\mathrm{N}$-terminal fragments of DnaE (DnaEc and DnaEn). A PEST sequence is attached at the C-terminal end to decompose the unspliced product. (b) Principle for monitoring activity of caspase-3 using cyclic firefly luciferase. 
a

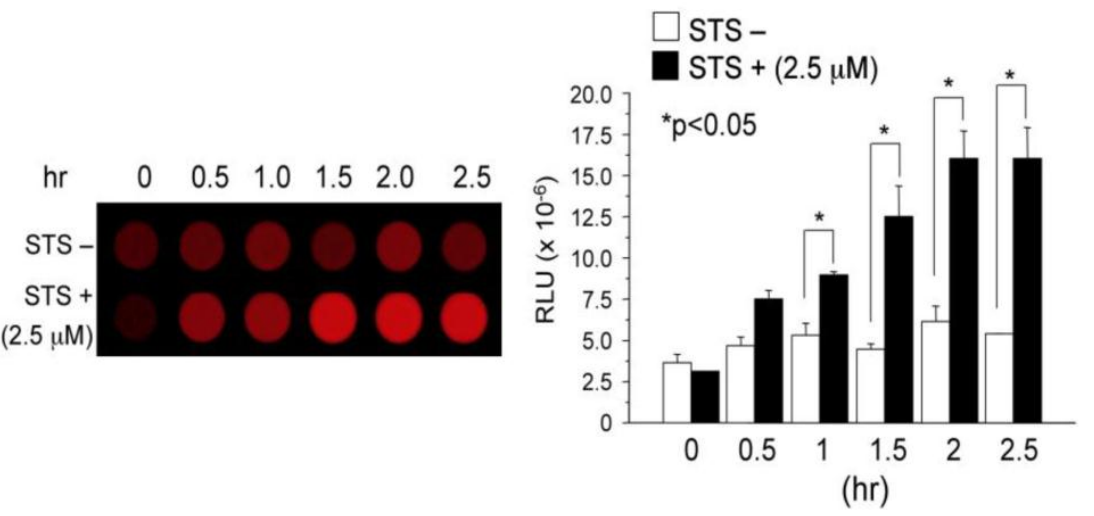

b
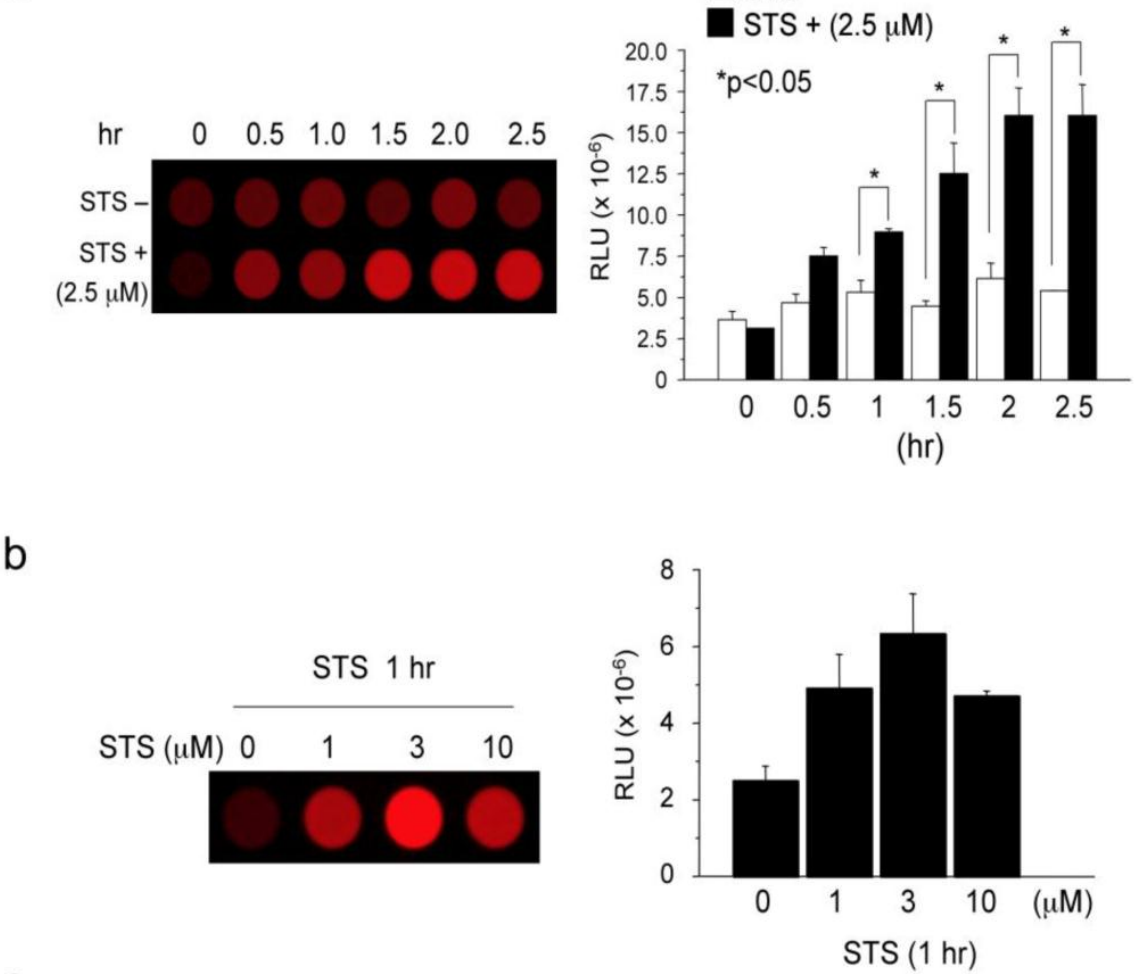

C

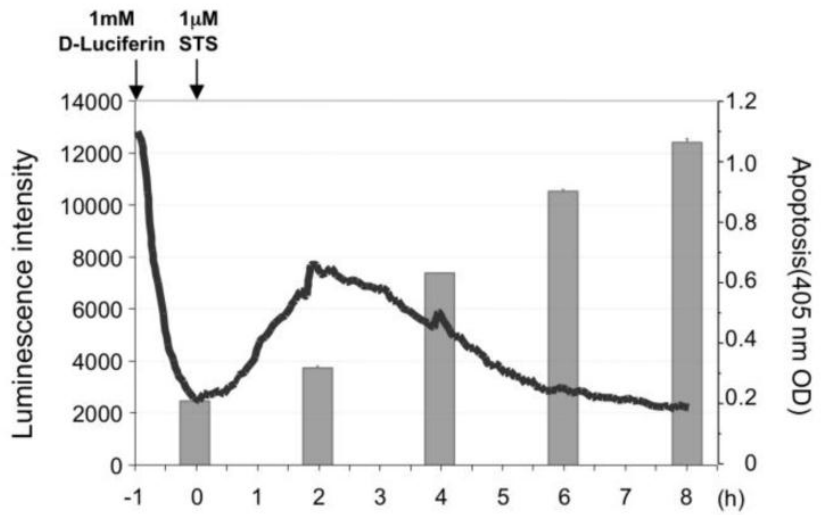

Figure 2. (a) Measurement of staurosporine-induced Caspase-3 activity: time course (activity in cell lysates). (b) Measurement of staurosporine-induced Caspase-3 activity: dose response (activity in cell lysates). (c) Comparison of staurosporine-induced Caspase-3 activity (live cells) and apoptosis. Results are expressed as mean \pm s.e.m. of five independent experiments. A $P$-value $<0.05$ was inferred as significant.

\section{In vitro measurement of cellular apoptosis in AMLI 2 liver cells challenged by staurosporine and Fas-ligand}

STS and Fas ligand respectively activate caspase-3 caspase-8-independently and dependently. First, we measured caspase- 3 activity in cell lysates prepared from STS-challenged AML12 cells that had been transiently transfected with caspase-3 probe (pcFluc-DEVD) (Fig. 2a). The $2.5 \mu \mathrm{M}$ STS immediately induced caspase- 3 activity and continued for at least $2.5 \mathrm{hr}$ (peak value at $2 \mathrm{hr}$ ), although untreated cells showed only basal level signals. In fact, 1, 3, and 10 $\mu \mathrm{M}$ of STS also induced caspase- 3 activity in AML12 liver cells, with a peak value at the concentration of 3 $\mu \mathrm{M}$ (Fig. 2b). Therefore, we checked the relations between STS-induced caspase-3 activity and apoptosis (Fig. 2c). Signals from the probe started to increase immediately after STS administration to AML12 cells. They peaked at $2 \mathrm{hr}$ and gradually returned to basal 
levels thereafter. The decrease in the luminescence after peaking at $2 \mathrm{hr}$ might originate from decomposition of the probe, decreasing the ATP or the substrate D-luciferin. In accordance with caspase-3 activation, cellular apoptosis occurred $4 \mathrm{hr}$ after STS-treatment and continued to increase until $8 \mathrm{hr}$ post-treatment. These data indicate the biological accuracy of caspase- 3 activity indicated by our probe.

Clinically, Fas has been shown to be important in hepatitis and other liver diseases [11-13]. The liver is extremely sensitive to Fas-mediated apoptosis because Fas antigen is expressed constitutively on hepatocytes. The Fas/FasL system is apparently in-

a

$$
\text { Jo-2 }(\mu \mathrm{g} / \mathrm{ml}) \quad 0 \quad \frac{6 \mathrm{hr}}{1.0} \quad \frac{12 \mathrm{hr}}{1.0} \quad \frac{2.0}{1.0}
$$

Cleaved caspase-3

b

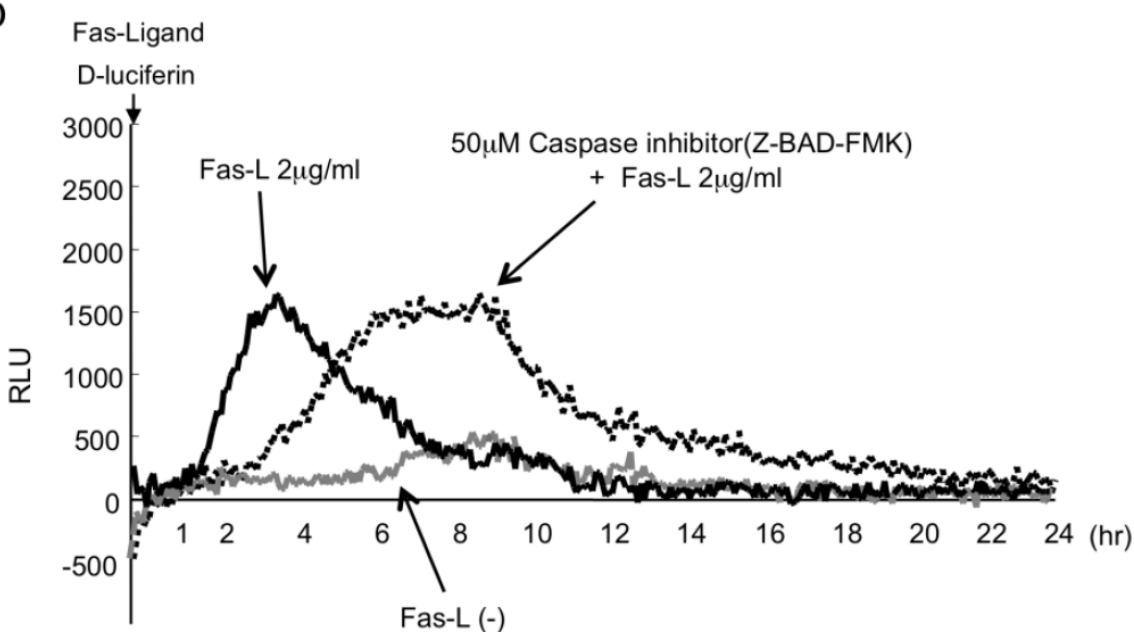

C

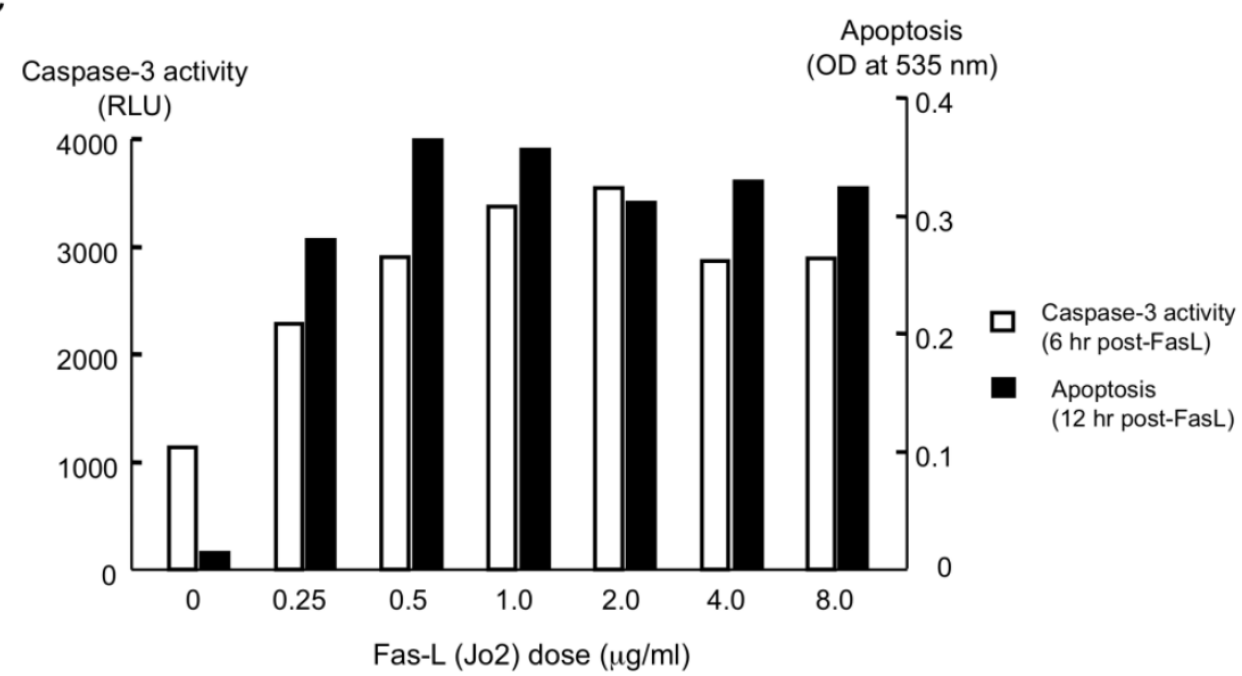

Figure 3. (a) Binding of Fas-ligand (Jo-2) to Fas-activated caspase-3 (increase of cleaved caspase-3). (b) Measurement of Fas-L-induced caspase-3 activity in live liver cells. (c) Comparison of FasL-induced caspase-3 activity (live cells) and apoptosis in AMLI 2 liver cells. 
It is particularly interesting that the peak values (the caspase- 3 activity intensity) were not suppressed by the administration of a general caspase inhibitor, Z-BAD-FMK, but were simply delayed about $4 \mathrm{hr}$, maintaining equal peak values. These data suggest that Z-BAD-FMK does not suppress caspase reactions: it merely retards the cascade reactions of caspases. To confirm the biological activity of caspase- 3 in FasL-challenged AML12 cells, we also studied apoptotic cell death as well as caspase- 3 activity. In Fas-L treated cells, caspase- 3 activity was induced immediately after the treatment and paralleled with apoptotic cell death (Fig. 3c).

\section{In vitro evaluation of cellular apoptosis by caspase-3 activity in post-hypoxic AMLI 2 liver cells}

During hypoxia of AML12 liver cells, the caspase-3 signals dropped to minimal levels because of rapid loss of cellular ATP (Fig. 4). The signals recovered rapidly to the basal levels after reoxygenation and rose until 4-6 hr post-H/R.

These data indicate that our probe is applicable to various biological systems. In the experiments described hereinafter, we applied this probe as a marker of apoptotic cell death in in vivo as well as in vitro experiments.

\section{In vivo evaluation of liver apoptosis by caspase-3 activity in hepatic ischemia/reperfusion (I/R) model}

Adenovirus vector encoding caspase- 3 probe (AdpcFluc-DEVD) was injected intravenously 3 days before the experiment $\left(5 \times 10^{7} \mathrm{pfu} /\right.$ body $)$. Liver ischemia was obtained by clamping all vessels (hepatic artery, portal vein, and bile duct) to the left and median liver lobes. After liver ischemia, these vessels were unclamped for hepatic reperfusion. Then D-luciferin, a luciferase substrate, was injected intraperitoneally at a dose of $3 \mathrm{mg} / 100 \mu \mathrm{l}$ in PBS. In vivo imaging of the mouse liver was performed using an in vivo imager for $5 \mathrm{~min}$, from 5-10 min after injection [15].

The pcFluc-DEVD probe indicated dynamic changes of liver damage chronologically and quantitatively by visualizing caspase-3 activity in the post-ischemic liver (Fig. 5a). Caspase-3 activity, i.e. cellular apoptosis, increased 2-6 hr after reperfusion of the liver and gradually decreased until $24 \mathrm{hr}$ in 30 -min post-ischemic liver. Actually, 60 -min ischemia showed a great increase of caspase- 3 activity (apoptotic liver damage) at the later time point (6-9 hr). It is particularly interesting that prolonged ischemia $(90$ min of ischemia) did not induce apoptotic cell death. Instead, necrotic cell death increased significantly, as assessed by serum levels of GPT / LDH (Fig. 5b).

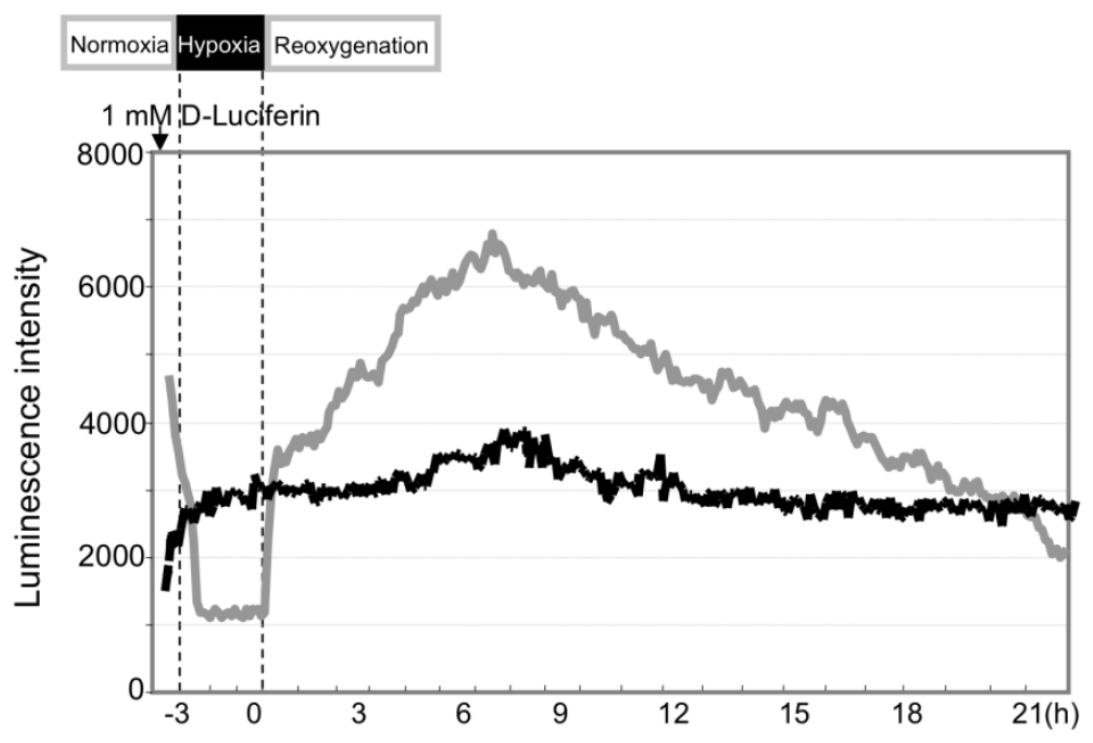

Figure 4. Hypoxic insult induced activation of caspase-3 in AMLI2 liver cells. Compared to the control cells without hypoxia (black line), optical signals dropped during hypoxia. They recovered to the baseline and rapidly increased after reoxygenation (gray line). 
a
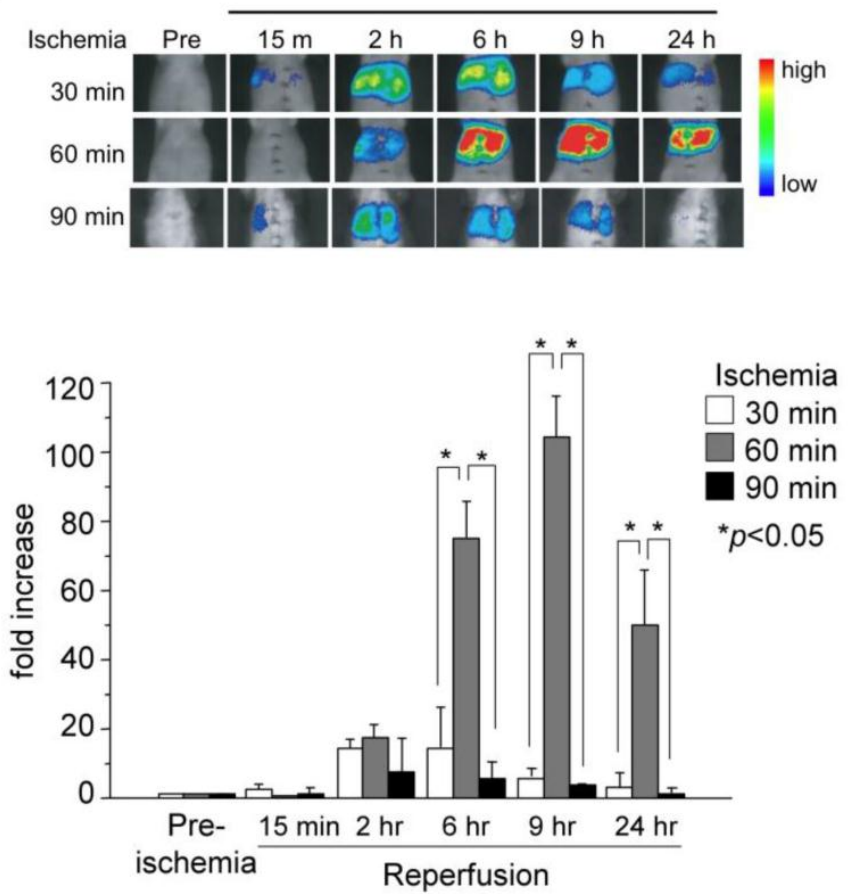

b
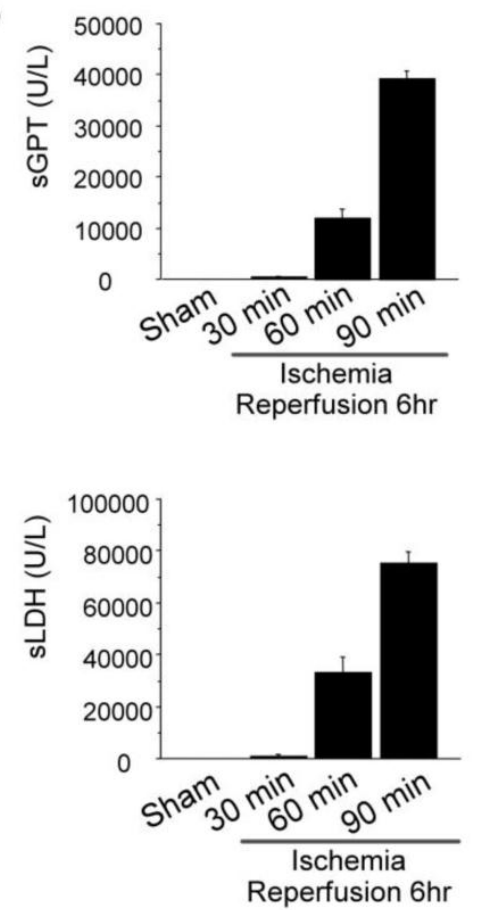

Figure 5. (a) Ischemic insult to liver induced caspase-3 activation. (It. and middle liver ischemia and reperfusion). (b) Serum levels of GPT and LDH (necrotic markers of liver) $6 \mathrm{hr}$ after hepatic I/R. At least three mice were used for each experiment. The photographs are representative of three independent experiments. Data are expressed as mean \pm s.e.m.

These data revealed time-strength relations of post-ischemic apoptotic damage in the liver, and also might provide more information and some pathological insight into I/R-induced liver damage. Longer liver ischemia might not simply give stronger damage, but persistent damage to the post-ischemic liver by continued activation of caspases (apoptosis) and/or by necrosis. However, prolonged ischemia induced cell death directly by necrosis, not by apoptosis.

\section{Discussion}

The optical probe for caspase- 3 activity probe detected cellular/organ apoptotic cell death in various conditions, which clearly illustrated liver damage by $I / R$ in mouse liver. The prolonged ischemia induced profound liver damage by persistent apoptotic damage with a peak at a later time point. These data undoubtedly present the possibility of these probes in the analysis and understandings of physiopathological conditions in vivo. In many surgical treatments such as cell/organ transplantation and surgical resection of the pathological organs, H/R or I/R are unavoidable events. Probes of this kind will definitely provide, intra-operatively and also post-operatively, important information of cell and organ conditions, allowing a prediction about the current cell and organ damage and viability and functional failure at later time points.

Bio-probes of these kinds showing apoptosis and other cellular phenomena/conditions 1) might provide useful tools to discover new drugs and to avoid their side effects, 2) might enable personalized drug therapy by showing drug effects in patients, and 3) might support selection of the best drugs for each patient by pre-checking drug effects ex vivo. Thereby, biological probes of this kind will possess potential clinical relevance as a new diagnostic and therapeutic tool. Caspase probes revealed apoptotic damage in vitro and in vivo. By showing cell and organ conditions, these might provide many options for future for future diagnoses.

\section{Acknowledgements}

This work was supported by the Japan Society for the Promotion of Science (JSPS), Japan Science and Technology Corporation (JST), a Grant-in-Aid for Scientific Research from the Ministry of Education, Culture, Sports, Science, and Technology of Japan (\# 20249060 and \#20249066 to M.O., \#26575001 to T.O.) 
and JSPS grant for Young Scientists (S.H.).

\section{Materials and Methods}

AMLI 2 liver cells, adenovirus vector (AdpcFluc) and reagents

Alpha mouse liver 12 (AML12) cells (ATCC, Manassas, VA), established from hepatocytes from a mouse transgenic for human transforming growth factor-a (TGF-a), express high levels of human TGF- $\alpha$ and lower levels of mouse TGF-a. Replication-deficient recombinant adenovirus-encoding pcFluc-DEVD (AdpcFluc) were generated in our laboratory and transfected into AML12 cells at five multiplicities of infection (moi) or injected to mice intravenously ( $5 \times 10^{7} \mathrm{pfu} /$ body $)$ at $48 \mathrm{hr}$ or $72 \mathrm{hr}$ before the experiment. To examine basic functions of the caspase- 3 optical probe, homogenates of AML12 cells $\left(2 \times 10^{5} / 3.5 \mathrm{~cm}\right.$ Dish $)$ challenged with staurosporine (STS) were applied for in vitro cell-free bio-luminescence activity assay. Briefly, 1-10 $\mu \mathrm{M}$ of STS was administered in AML12 cells. Cells were harvested sequentially and their caspase-3 activity was measured (Bright-Glo Luciferase Assay System, GloMax®-20/20 Luminometer; Promega Corp.). D-luciferin K salt was purchased (Dojindo Laboratories, Japan). All other chemicals were of analytical grade and were used without further purification.

\section{Measurements of bio-luminescence activity for caspase- 3 in live cells and apoptosis challenged by $H / R$ or Fas ligand (FasL)}

To obtain cellular hypoxic conditions, cells were ventilated by continuous gas flow with a $95 \% \mathrm{~N}_{2}=5 \%$ $\mathrm{CO}_{2}$ gas mixture $(150 \mathrm{ml} / \mathrm{min})$. This method has been shown to achieve a $\mathrm{pO}_{2}$ of $10 \times 5$ Torr (where 1 Torr. $0.133 \mathrm{kPa}$ ) within $2 \mathrm{~min}$. After hypoxia of AML12 cells, reoxygenation was achieved by flushing continuously with a $95 \%$ air $/ 5 \% \mathrm{CO}_{2}$ gas mixture. After adding D-luciferin substrate $(1 \mathrm{mM})$, bioluminescence for caspase-3 activity in live cells during $H / R$ or stimulated by FasL was measured chronologically (Kronos-Dio; Atto Corp., Tokyo, Japan). Apoptotic cell death was measured using a kit (Cell Death Elisa; Roche, Switzerland) according to the manufacturer's instructions to ensure the accuracy of this probe as an apoptotic marker.

\section{In vivo imaging of mouse liver challenged with $\mathbf{I} / \mathbf{R}$}

Male C57Black/6 mice, 8-10 weeks old, were starved overnight before use. Three days before each experiment, adenovirus vector coding for pcFluc-DEVD (Ad pcFluc-DEVD) was administered intravenously (iv) via the tail vein in a volume of 100 $\mu \mathrm{l}\left(5 \times 10^{7} \mathrm{pfu} /\right.$ body $)$ using a $31 \mathrm{G}$ needle. General anesthesia was induced with the inhalation anesthetic methoxyflurane (Metofane); heparin sulfate (100 $\mathrm{U} / \mathrm{kg}$ body weight) was injected intravenously. After laparotomy, all vessels (hepatic artery, portal vein, and bile duct) to the left and median liver lobes were clamped. After 30-60 min of liver partial ischemia, these vessels were unclamped. Then the hepatic circulation was restored for the specified reperfusion period. D-luciferin was injected intraperitoneally at a dosage of $3 \mathrm{mg} / 100 \mu \mathrm{l}$ in PBS. In vivo imaging of the mouse liver was performed using an in vivo imager for $5 \mathrm{~min}$ from $5-10 \mathrm{~min}$ after injection by a Photon Imager (Biospace Lab, Paris, France).

\section{Statistical Analysis}

Results were expressed as mean \pm s.e.m. Statistical analyses were performed using Fisher's test; $P$-values $<0.05$ were considered significant.

\section{Conflict of Interest}

The authors have declared that no conflict of interest exists.

\section{References}

1. Dooley CT, Dore TM, Hanson GT et al. Imaging dynamic redox changes in mammalian cells with green fluorescent protein indicators. J Biol Chem. 2004; 279: 22284-93.

2. Kanno A, Yamanaka $\mathrm{Y}$, Hirano $\mathrm{H}$ et al. Cyclic luciferase for real-time sensing of caspase-3 activities in living mammals. Angew Chem Int Ed. 2007; 46: 7595-9.

3. Zhu L, Xie J, Swierczewska M et al. Real-time video imaging of protease expression in vivo. Theranostics. 2011; 1: 18-27.

4. Lexman B, Hall DE, Bhojani MS et al. Noninvasive real-time imaging of apoptosis. Proc Natl Acad Sci U S A. 2002; 99: 16551-5.

5. Ray P, De A, Patel M, Gambhir SS. Monitoring caspase-3 activation with a multimodality imaging sensor in living subjects. Clin Cancer Res. 2008; 14: 5801-9.

6. Yhee JY, Kim SA, Koo H et al. Optical imaging of cancer-related proteases using near-infrared fluorescence matrix metalloproteinase-sensitive and cathepsin B-sensitive probes. Theranostics. 2012; 2: 179-89.

7. Kim GB, Kim Y.-P. Analysis of protease activity using quantum dots. Theranostics. 2012; 2: 127-38.

8. Ozaki M, Deshpande SS, Angkeow P et al. Inhibition of the Rac1 GTPase protects against nonlethal ische$\mathrm{mia} /$ reperfusion-induced necrosis and apoptosis in vivo. Faseb J. 2000; 14: 418-29.

9. Lucchesi, BR. Complement activation, neutrophils, and oxygen radicals in reperfusion injury. Stroke. 1993; 24: I41-7.

10. Haga S, Remington S, Morita $N$ et al. Hepatic ischemia induced immediate oxidative stress after reperfusion and determined the severity of the reperfusion-induced damage. Antioxid Redox Signal. 2009; 11: 2563-72.

11. Pinkoski MJ, Brunner T, Green D.R et al. Fas and Fas ligand in gut and liver. Am J Physiol Gastrointest Liver Physiol. 2000; 278: G354-66.

12. Galle PR, Hofmann WJ, Walczak $\mathrm{H}$ et al. Involvement of the CD95 (APO-1/Fas) receptor and ligand in liver damage. J Exp Med. 1995; 182: 1223-30. 
13. Kanzler S, Galle PR. Apoptosis and the liver. Semin Cancer Biol. 2000; 10: 173-84.

14. Faubion WA, Guicciardi ME, Miyoshi $\mathrm{H}$ et al. Toxic bile salts induce rodent hepatocyte apoptosis via direct activation of Fas. J Clin Invest. 1999; 103: 137-45.

15. Haga S, Morita N, Irani $\mathrm{K}$ et al. p66 ${ }^{\text {Shc }}$ plays a pivotal role in impaired liver regeneration in aged mice by a redox-dependent mechanism. Lab Invest. 2010; 90: 1718-26. 